University of Nebraska - Lincoln

DigitalCommons@University of Nebraska - Lincoln

Faculty Publications, Department of Physics and Astronomy

Research Papers in Physics and Astronomy

2008

\title{
Theory of the Piezomagnetic Effect in Mn-Based Antiperovskites
}

\author{
Pavel Lukashev \\ University of Nebraska-Lincoln, pavel.lukashev@uni.edu \\ Renat F. Sabirianov \\ University of Nebraska at Omaha, rsabirianov@mail.unomaha.edu \\ Kirill D. Belashchenko \\ University of Nebraska-Lincoln, belashchenko@unl.edu
}

Follow this and additional works at: https://digitalcommons.unl.edu/physicsfacpub

Part of the Physics Commons

Lukashev, Pavel; Sabirianov, Renat F.; and Belashchenko, Kirill D., "Theory of the Piezomagnetic Effect in Mn-Based Antiperovskites" (2008). Faculty Publications, Department of Physics and Astronomy. 72. https://digitalcommons.unl.edu/physicsfacpub/72

This Article is brought to you for free and open access by the Research Papers in Physics and Astronomy at DigitalCommons@University of Nebraska - Lincoln. It has been accepted for inclusion in Faculty Publications, Department of Physics and Astronomy by an authorized administrator of DigitalCommons@University of Nebraska Lincoln. 


\title{
Theory of the piezomagnetic effect in Mn-based antiperovskites
}

\author{
Pavel Lukashev, ${ }^{1}$ Renat F. Sabirianov, ${ }^{2}$ and Kirill Belashchenko ${ }^{1}$ \\ ${ }^{1}$ Department of Physics and Astronomy, University of Nebraska-Lincoln, Lincoln, Nebraska 68588, USA \\ ${ }^{2}$ Department of Physics, University of Nebraska-Omaha, Omaha, Nebraska 68188, USA
}

(Received 12 May 2008; revised manuscript received 11 September 2008; published 14 November 2008)

\begin{abstract}
Recent experimental and theoretical studies of the magnetoelectric (ME) effect in the nanocomposite structures and in laminates show an enhanced ME coefficient. These materials combine piezoelectric properties of the paramagnetic phase and piezomagnetic properties of the magnetic phase. We propose to fabricate heterostructures formed by piezoelectric materials and magnetic antiperovskites as magnetoelectric materials. We show that the magnetic structure of antiperovskite, such as $\mathrm{Mn}_{3} \mathrm{GaN}$, can be controlled by a small applied biaxial strain. The lowering of symmetry with the strain causes the local magnetic moments of $\mathrm{Mn}$ atoms to rotate from the trigonal $\Gamma^{5 g}$ structure with symmetric curl of spin density in the (111) plane to a monoclinic symmetry structure. As a result, an appreciable net magnetization appears in the strained system.
\end{abstract}

DOI: 10.1103/PhysRevB.78.184414

PACS number(s): 75.80.+q, 75.50.-y, 77.65.-j, 77.84.Lf

Materials and nanostructures with strong coupling between magnetic and electric order parameters and/or fields are of great interest for practical applications. ${ }^{1}$ In particular, the prospect of inducing a magnetic moment by external electric field is promising for the design of novel magnetoelectronic devices. ${ }^{2}$ Although some antiferromagnets, such as $\mathrm{Cr}_{2} \mathrm{O}_{3}$, possess an intrinsic linear magnetoelectric (ME) effect, they are uncommon, and the magnitude of the effect is quite small. Therefore, much research is focused on the design of two-phase heterostructures where the electric polarization and the magnetic moment are intrinsic to the two different phases, but coupled by interfacial strain. Usually the magnetic phase in such a heterostructure is ferromagnetic, and its response to strain is through magnetostriction, which alters the magnetocrystalline anisotropy and hence the hysteresis properties.

In this paper we consider an alternative approach using a piezomagnetic (PM) material instead of a magnetostrictive one. Piezomagnetic materials are also uncommon, the textbook example being the hematite $\mathrm{Fe}_{2} \mathrm{O}_{3}$. It is fruitful to search for piezomagnetic materials among noncollinear antiferromagnets because noncollinearity usually implies magnetic frustration and competition between different magnetic orderings. Such materials may be strongly piezomagnetic because the response to stress may involve rotations of the magnetic moments. Here we consider a noncollinear antiferromagnet $\mathrm{Mn}_{3} \mathrm{GaN}$ with an antiperovskite structure. An additional advantage of these (and similar) materials is that the crystal structure is very well matched to perovskite ferroelectrics and piezoelectrics which may be used to provide controllable epitaxial strain in a heterostructure.

Magnetic antiperovskites show a variety of magnetic orderings and phase transitions. These materials exhibit superconductivity with critical temperature of $8 \mathrm{~K}\left(\mathrm{Ni}_{3} \mathrm{MgC}\right)$ (Ref. 3) and a giant magnetoresistance of more than $10 \%$ in pulsed magnetic fields $\left(\mathrm{Mn}_{3} \mathrm{GaC}\right) .{ }^{4}$ Due to the interplay of magnetic and elastic properties, $\mathrm{Mn}_{3} A \mathrm{~N}(A=\mathrm{Ga}, \mathrm{Zn}, \mathrm{Cu})$ exhibits the invar effect (or even negative thermal expansion) ${ }^{5}$ and a near zero temperature coefficient of resistance. ${ }^{7}$ Many of these properties are related to the first-order "cubic-tocubic" phase transition where the spin structure changes from noncollinear to paramagnetic. ${ }^{8}$ Recent studies show that doping changes the transition temperature of antiperovskites and modifies the phase transition from first-order to second-order. 5

The ground state of $\mathrm{Mn}_{3} A \mathrm{~N}$ is shown in Fig. 1. This is a noncollinear, $\Gamma^{5 g}$ in the classification of Bertaut et al., ${ }^{14}$ structure with the spins on the (111) plane with clockwise or counterclockwise spin-density configurations, such that the spin moments in each plane cancel each other. The noncollinearity in this compound appears due to the antiferromagnetic (AFM) coupling between spins of nearest Mn atoms. Due to the frustration in triangular lattice in (111) plane the lowest-energy magnetic structure has $120^{\circ}$ angle between the directions of local magnetic moments (LMMs). Spin-orbit (SO) interactions govern the particular orientation of such noncollinear structure with respect to the lattice, i.e., the direction of easy axis. ${ }^{6}$ In case of unstrained cubic lattice planes, the magnetization curl is perpendicular to (111) axis and the spin chiralities in (111) planes are alternating along the [111] direction. ${ }^{8}$

In this paper we report on the PM effect in $\mathrm{Mn}_{3} \mathrm{GaN}$, i.e., on the appearance of the net magnetization upon application of in-plane biaxial strain in the (001) plane. This strain can be experimentally obtained by growing thin films on the substrate with larger or smaller lattice parameters. We also discuss forming antiperovskite-perovskite ferroelectric heterostructures and applying an external voltage. These heterostructures may exhibit a large magnetoelectric effect.

We employ the projector augmented-wave (PAW) method originally proposed by Blöchl. ${ }^{9}$ We use the implementation of Kresse and Joubert in VASP code ${ }^{10}$ within the PerdewBurke-Ernzerhof (PBE) generalized gradient approximation $^{11}$ for fully unconstrained noncollinear magnetic structures. ${ }^{12} \mathrm{We}$ use a $12 \times 12 \times 12 k$-point sampling and Blöchl's tetrahedron integration method. ${ }^{13}$ The cutoff energy is $300 \mathrm{eV}$. We calculate the total energy, magnetic moments, densities of states (DOSs), and related properties for the noncollinear $\Gamma^{5 g}$ and the AFM magnetic phases as a function of the volume. The AFM phase is constructed to have three mutually perpendicular antiferromagnetic Mn-N-Mn chains along (100) directions. This AFM structure has zero magnetization and mimics a high-temperature paramagnetic phase. We have applied a biaxial strain in the (001) plane and 

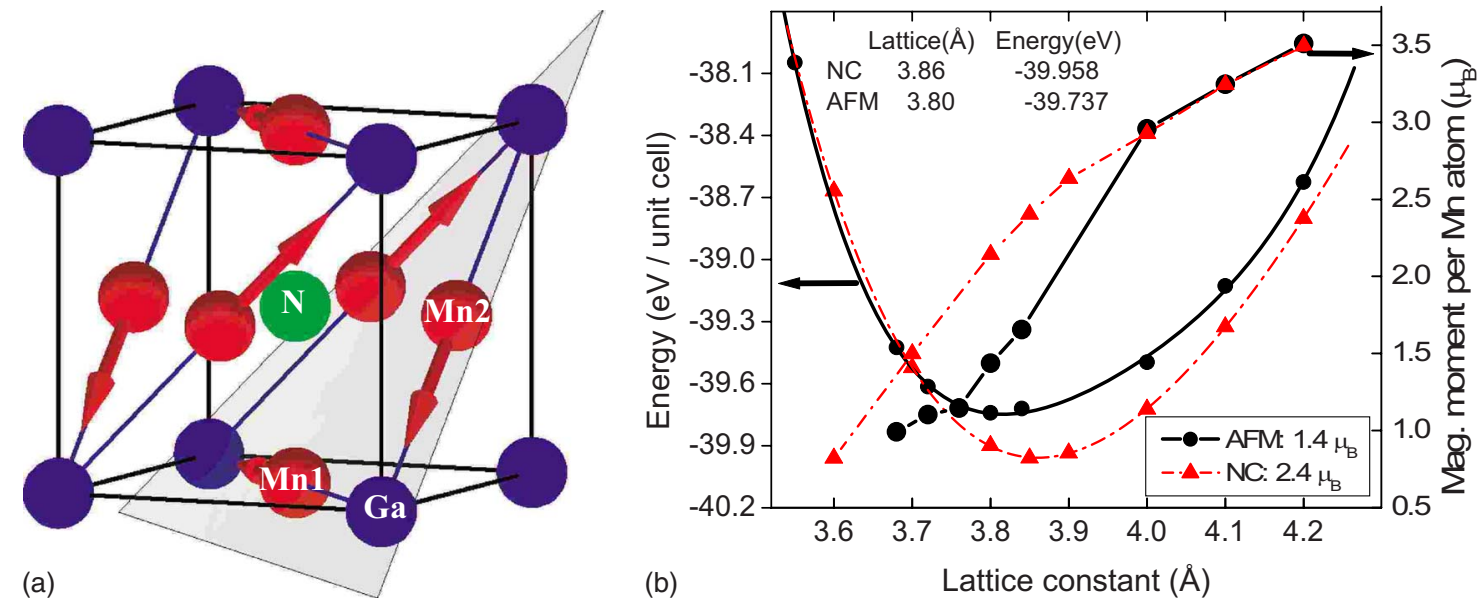

FIG. 1. (Color online) (a) Noncollinear $\Gamma^{5 g}$ structure, unit cell. (b) Energy and local magnetic moment of Mn atom as functions of the lattice constant for $\mathrm{Mn}_{3} \mathrm{GaN}$ in $\Gamma^{5 g}$ and $\mathrm{AFM}$ structures.

relaxed the shape of the cell as well as the atomic positions.

Noncollinear $\Gamma^{5 g}$ is the ground-state structure for $\mathrm{Mn}_{3} \mathrm{GaN}$ and the calculated equilibrium lattice constant is $3.86 \AA$ compared with the experimental value of $3.898 \AA$ (at $273 \mathrm{~K}) .{ }^{14}$ The calculated LMM integrated over the volume of the space-filling atomic sphere (1.769 $\AA$ for Mn sites) is $2.4 \mu_{B}$ at its equilibrium lattice parameter as shown in Fig. 1. The local magnetic moment is equal to $1.4 \mu_{B} / \mathrm{Mn}$ atom in AFM structure at its equilibrium lattice constant, about half of that for $\Gamma^{5 g}$ structure. The total energy of the AFM structure is $0.22 \mathrm{eV} / \mathrm{f}$.u. higher than that of $\Gamma^{5 g}$ structure at their optimal lattices. The equilibrium lattice constant for AFM phase is $3.80 \AA$ and it is smaller than that in $\Gamma^{5 g}$ structure by $0.06 \AA(\Delta a / a=0.015)$. This agrees with the observed $\Gamma^{5 g}$ $\rightarrow$ paramagnetic first-order phase transition close to the room temperature which is accompanied by a lattice contraction of $\Delta a / a=0.004$. The calculated value is somewhat larger because calculations are performed at zero temperature. If experimentally observed dependences of lattice parameter in two phases extend to zero temperature, $\Delta a / a$ would be about 0.010 which is in reasonable agreement with the calculated value. $^{5}$

Experimentally $\mathrm{Mn}_{3} \mathrm{GaN}$ may have volumes different from equilibrium, for example, if this compound appears as a precipitate in Mn-doped GaN. ${ }^{15}$ Figure 1 shows that the $\mathrm{Mn}$ local magnetic moment is extremely sensitive to the volume change. In the $\Gamma^{5 g}$ structure it steadily increases from $1 \mu_{B}$ to $3.5 \mu_{B}$ as the lattice parameter increases from $3.6 \AA(18 \%$ volume compression) to $4.2 \AA$ ( $30 \%$ volume expansion). In the AFM phase the LMM also increases with volume expansion with somewhat sharper transition from the low-spin state to the high-spin state. This is connected with the filling of the minority band which has a large peak of DOS just below the Fermi energy.

There are no recent neutron diffraction data on the magnetic moments of $\mathrm{Mn}_{3} \mathrm{GaN}$. Original measurements by Fruchart and Bertaut suggested that the local magnetic moment on a Mn site is $1.17 \mu_{B} .{ }^{8}$ However, in this work Fruchart and Bertaut measured the magnetic moment for the whole series of $\mathrm{Mn}_{3} A \mathrm{~N}$ and they found similar moments for most of the compounds including $\mathrm{Mn}_{3} \mathrm{ZnN}\left(1.21 \mu_{B}\right)$. Yet, recent neutron diffraction measurements have shown a larger magnetic moment of about $2.1 \mu_{B}$ for $\mathrm{Mn}_{3} \mathrm{ZnN}$. $^{16}$ This is supported by our $a b$ initio analysis (the calculated magnetic moment is near $\left.2.4 \mu_{B}\right)$. In view of this development we think that neutron measurements for the whole series should be re-examined to resolve this situation. Our calculations as well as previously reported results ${ }^{17}$ show magnetic moment of the order of $2.4 \mu_{B}$ in agreement with more recent experimental data. ${ }^{16}$ The value of the local magnetic moment is not uniquely defined in calculations and depends on the choice of the radius of the projection sphere. For our system, it is not very sensitive to the choice of the projection sphere radius around space filling scheme. For example, LMM value for the touching sphere case (radius of the projection sphere is $1.46 \AA$ ) is $2.34 \mu_{B}$.

Although $\mathrm{Mn}_{3} \mathrm{GaN}$ does not possess a spontaneous magnetization, it may develop one under mechanical stress, $\sigma{ }^{18}$ This is the phenomenon of piezomagnetism and it is characterized by a piezomagnetic tensor $q\left(\mu_{0} M=q \sigma\right)$. The elements of this tensor are nonzero for 66 piezomagnetic crystal classes including $\overline{3} m$ and $2 / m .^{19}$ The transition from the paramagnetic structure to the $\Gamma^{5 g}$ noncollinear structure is frequently described as a rare cubic-to-cubic phase transition. This is true only if the magnetic symmetry is omitted from the consideration. The undistorted $\mathrm{Mn}_{3} \mathrm{GaN}$ belongs to the trigonal space group $P \overline{3} 1 \mathrm{~m}\left(D_{3 d}^{1}\right)$, and its symmetry is lower than cubic because of its noncollinear spin configuration. This classical space group can be derived from a cubic Pm3m space group, which this system belongs to (in the paramagnetic phase), by inspection of representations of this group. The $\mathrm{Mn}_{3} \mathrm{GaN}$ symmetry corresponds to $\Gamma^{5 g}$ representation. ${ }^{14}$ The following symmetry operations can be chosen as the group generating elements in an unstrained cell: $\{I \mid 000\},\left\{C_{3} \mid 000\right\},\left\{S_{6} \mid 000\right\},\left\{\sigma_{d 1} \mid 000\right\}$, and $\left\{C_{21} \mid 000\right\}$. As a result of the applied biaxial strain the number of possible symmetry operations in the system changes. After a biaxial strain is applied, a rotation of the third order is not compatible with the lattice, and this reduces the symmetry to the orthorhombic $P m^{\prime} m^{\prime} m$ ferromagnetic space group with the following generating elements: $\{I \mid 000\},\left\{C_{2 z} \mid 000\right\}$, $\left\{\sigma_{z} \mid 000\right\}$, and $\left\{C_{2 x} \mid 000\right\} \theta,\left\{\sigma_{x} \mid 000\right\} \theta$, where the latter two 


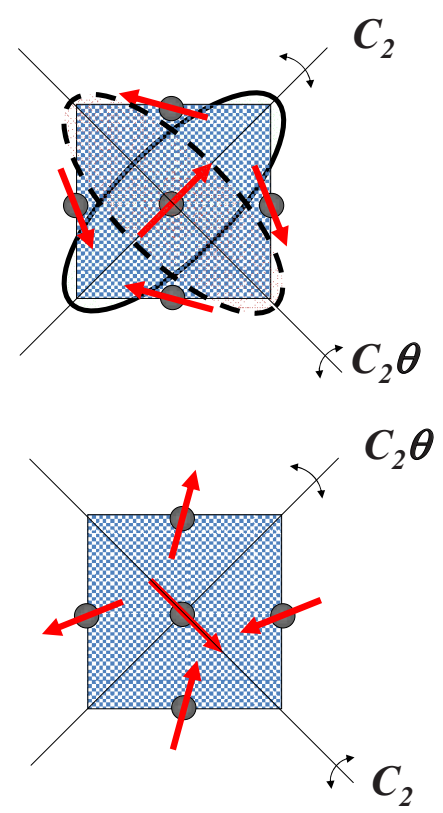

FIG. 2. (Color online) The noncollinear $\Gamma^{5 g}$ magnetic structure of strained $\mathrm{Mn}_{3} \mathrm{GaN}$ with magnetization along (110) (upper panel) and the spin configuration obtained by continuous rotation of magnetization to (110) (lower panel). The solid curve in the upper panel shows the change in magnetocrystalline energy (MAE) as a function of the direction of net magnetization upon its rotation. The dashed curve shows MAE for domain with magnetization along (100).

have an antisymmetry (time-reversal) operation, $\theta$. In the latter case, the plane of the curl of the magnetic spin density is no longer along (111) direction but shifts to the (001) direction because of the symmetry lowering and the absence of the threefold symmetry axis. In case of biaxially strained lattice (111) is not a symmetry axis anymore. Thus, any infinitesimally small spin-orbit coupling should move the easy axis to the new high-symmetry direction. The consequence of such rearrangement is quite surprising. The continuous rotation of magnetization from $(110)$ to $(\overline{1} 10)$ direction results in inequivalent magnetic structure as shown in Fig. 2. If spin-orbit interactions are taken into account the energy of the modified structure is higher. On the other hand, crystallographically (110) and (110) axes are equivalent. This results in double-valued magnetocrystalline anisotropy which is not commonly observed in bulk systems (see Fig. 2). Experimentally it may be observed by asymmetric reversal astroid (or biaxial astroid) as a function of the angle of applied magnetic field. The system should exhibit a two-level noise in magnetization reversal process.

We have examined the modifications of the structural and electronic properties with the biaxial strain. The biaxial strain can be experimentally produced by growing the antiperovskite film on the substrate with a different lattice constant or piezoelectric (PE) substrate. The biaxial strain in (001) plane applied to $\mathrm{Mn}_{3} \mathrm{GaN}$ lowers its crystalline (paramagnetic phase) symmetry from cubic to tetragonal. The unit cell has a $c / a$ ratio less than 1 in case of the tensile biaxial strain and larger than 1 in case of the compressive strain. The

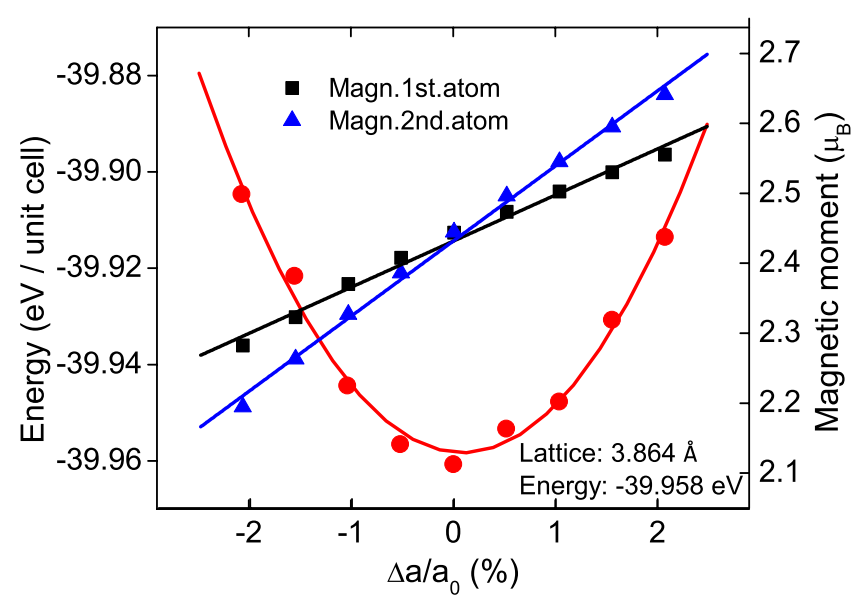

FIG. 3. (Color online) Energy (circles) and magnetic moments of Mn1 (squares) and $\mathrm{Mn} 2$ (triangles) atoms in $\mathrm{Mn}_{3} \mathrm{GaN}$ ( $\Gamma^{5 g}$ structure) as a function of strain.

Poisson ratio, $\nu$, is calculated from the ratio of the relative change in $c$-axis length, $\Delta c / c_{0}$, to the biaxial strain as follows:

$$
\frac{\Delta c / c_{0}}{\Delta a / a_{0}}=-\frac{2 \nu}{1-\nu} .
$$

Our calculated value of $\nu$ for $\mathrm{Mn}_{3} \mathrm{GaN}$ is $\nu=0.4$. Figure 3 shows the variation in the net magnetic moment per $\mathrm{Mn}$ atom for the $\Gamma^{5 g}$ structure as biaxial strain is applied.

Because of the large Poisson ratio the volume of $\mathrm{Mn}_{3} \mathrm{GaN}$ under biaxial tensile strain does not change significantly and the modification of the electronic and magnetic structure is connected with the lowering of the symmetry. We have relaxed the atomic positions in a supercell starting from a slightly distorted structure. In all our calculations the system retains tetragonal symmetry. No bond buckling or distortion is observed in the strained systems. The local magnetic moments are modified when the biaxial strain is applied, reflecting the modification in the crystalline symmetry. The LMMs in (111) plane become crystallographically inequivalent. In tetragonal unit cell two Wyckoff positions are occupied by manganese atoms: $1 b$ with the $\mathrm{Mn} 1$ atom in the basal plane (formed by atoms of $\mathrm{Mn}$ and $\mathrm{Ga}$ ) and $2 c$ with two Mn2 atoms in the $\mathrm{Mn}_{2} \mathrm{~N}$ plane. As one can see from Fig. 4 the magnetic moment of Mn1 preserves its direction, but its magnitude decreases upon compressive and increases upon tensile strain. LMMs of the Mn2 sites change both their direction and magnitude. LMMs of $\mathrm{Mn} 2$ sites rotate toward the [110] direction when the compressive strain is applied in such a way that it becomes almost opposite to LMM of Mn1 atom at large strains. The LMM of Mn2 sites becomes more aligned with each other (see Fig. 4). When the tensile strain is applied the LMMs of Mn2 sites rotate in direction opposite to the one at the compressive strain case. The Mn moments become more antialigned in this case as shown in Fig. 4. The magnitude of the LMMs generally decreases with the compressive strain and increases with the tensile strain as can be seen from the length of vectors in Fig. 4.

Thus, $\mathrm{Mn}_{3} \mathrm{GaN}$ antiperovskite acquires a net magnetiza- 


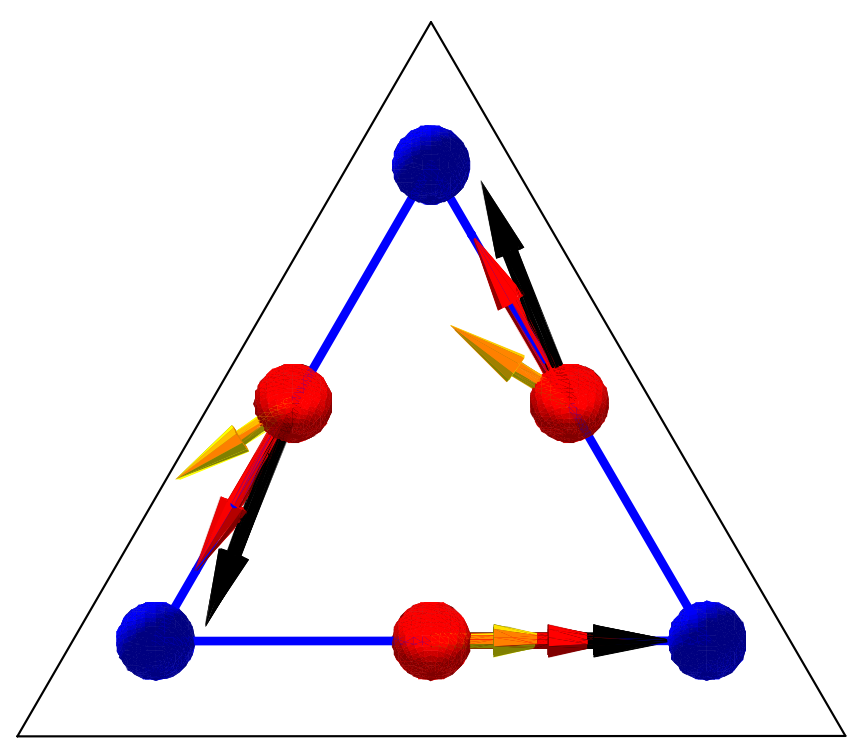

FIG. 4. (Color online) Variation in $\mathrm{LMM}$ of $\mathrm{Mn}_{3} \mathrm{GaN}$ in (111) plane of $\Gamma^{5 g}$ structure as a function of strain. LMMs for an unstrained lattice are directed along the lines connecting neighboring atomic sites (red arrows online), short arrows (yellow online) correspond to a compressive strain, long arrows (black online) correspond to a tensile strain.

tion under biaxial strain as a result of rotation of the direction of local magnetic moments and the change in their magnitude. The rotation angle of the LMM of a Mn2 atom under biaxial strain with respect to the Mn moment direction without strain as a function of the strain is shown in Fig. 5. The net magnetization which appears as a result of this rotation is directed along the $C_{2 z}$ axis (this corresponds to a diagonal axis $C_{2 a}$ in the cubic cell). Figure 5 shows the magnetization per cell (circles) as a function of the biaxial strain. The strain induced magnetization is quite large. For example, magnetization is about $0.04 \mu_{B} /$ f.u. at $1 \%$ of compressive strain. This piezomagnetic effect in $\Gamma^{5 g}$ antiperovskites is linear and exhibits magnetization reversal with the applied strain, i.e., the direction of the magnetization along the $C_{2 z}$ axis at the applied tensile biaxial strain reverses with the direction of the applied strain. This effect can be utilized for electrical control of the magnetization in memory cells. The piezomagnetic coefficient calculated from this graph for small strain is about $q=3 \times 10^{-10} \mathrm{Oe}^{-1}$.

In composite materials, the ME effect is realized by using the concept of product properties. A combination of two phases in the heterostructure can produce a large ME effect, as in the case of a combination of piezomagnetic and piezoelectric phases or a combination of magnetostrictive and piezoelectric phases. The latter combination may produce a magnetoelectric coefficient more than a hundred times that of single-phase $\mathrm{ME}$ materials such as $\mathrm{Cr}_{2} \mathrm{O}_{3} .{ }^{20}$ These laminate structures use the magnetostrictive effect. The strain caused by a magnetic field in these materials is not linearly proportional to the field strength but is related to the square of the magnetic field strength. This makes the applications of

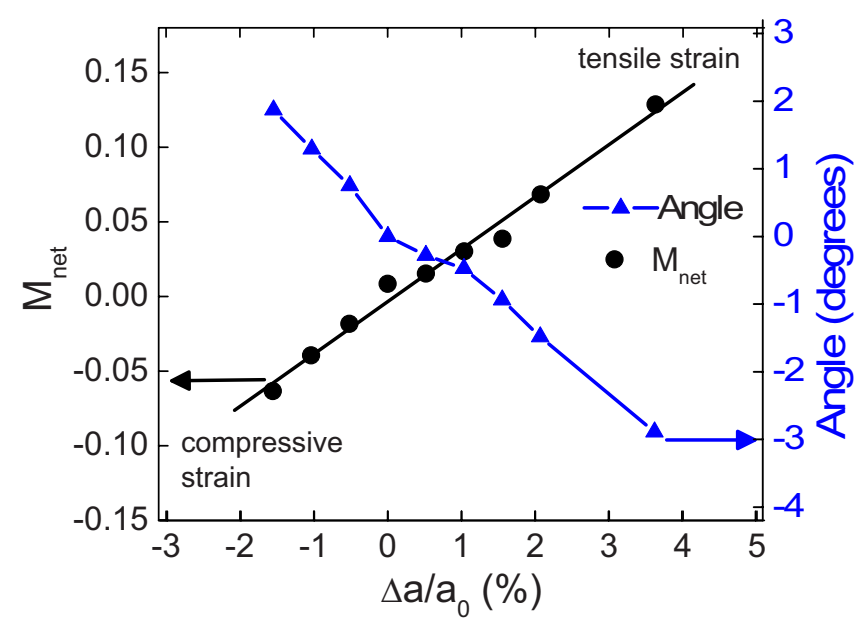

FIG. 5. (Color online) The net magnetic moment per unit cell and the rotation angle of $\mathrm{Mn} 2$ sites as functions of the biaxial strain $[\Delta a / a(\%)]$ in $\mathrm{Mn}_{3} \mathrm{GaN}$, noncollinear $\Gamma^{5 g}$ structure.

such composites difficult in linear devices. The piezomagnetic effect is linear and has been observed in several antiferromagnetic materials including $\mathrm{CoF}_{2}, \mathrm{MnF}_{2}$, and hematite.

The magnetoelectric coefficient is defined as $\alpha=\left(\frac{\delta P}{\delta H}\right)_{E}$ $=\left(\frac{\delta B}{\delta E}\right)_{H}$ and is measured in $\mathrm{s} / \mathrm{m}$ (or $\left.\mathrm{G} \mathrm{cm} / \mathrm{V}\right) \cdot{ }^{21}$ Assuming optimistic values of parameters in the two-phase composite resulting in $0.03 \mu_{B}$ change in the magnetic moment per unit cell $(\delta B=61 \mathrm{G})$ upon polarization, the average ME coefficient is about $0.001 \mathrm{G} \mathrm{cm} / \mathrm{V}$, which corresponds to $\alpha=1$ $\times 10^{-9} \mathrm{~s} / \mathrm{m}\left(\mathrm{Cr}_{2} \mathrm{O}_{3}\right.$ has 3 orders of magnitude lower value of $\left.\alpha=2.67 \times 10^{-12} \mathrm{~s} / \mathrm{m}\right)$. We assumed about $1 / 1$ ratio of PM and $\mathrm{PE}$ components and about $1 \%$ strain at the electric field of $30 \mathrm{kV} / \mathrm{cm}$ (typical for an upper end relaxor ferroelectric films ${ }^{22}$ ). This ME coefficient is large and comparable to recently reported measurements in epitaxial $\mathrm{BiFeO}_{3} / \mathrm{CoFe}_{2} \mathrm{O}_{4}$ nanocomposites. ${ }^{23} \mathrm{We}$ are not aware of any experimental studies of $\mathrm{Mn}_{3} \mathrm{GaN}$ films grown on oxides with perovskite structures, but we hope that our findings would stimulate such studies. Similar antiperovskite $\mathrm{Mn}_{3} \mathrm{GaC}$ film has been successfully epitaxially grown on perovskite $\mathrm{LaAlO}_{3}$ substrate. ${ }^{24}$ Although we presented results for a particular case of a biaxial strain, a similar effect is expected in the case of any strains which reduces the symmetry to be compatible with the piezomagnetism. For example, an anisotropic strain on a uniaxial substrate produces different strains along each axis. In this case we still expect appearance of the net magnetization upon applied strain due to similar reduction of symmetry.

\section{ACKNOWLEDGMENTS}

We thank Daniel Litvin for productive discussions. This work was supported by the Nebraska Research Initiative, the National Science Foundation, and the Nanoelectronics Research Initiative through the Materials Research Science and Engineering Center at the University of Nebraska. 
${ }^{1}$ W. Eerenstein, N. D. Mathur, and J. F. Scott, Nature (London) 442, 759 (2006).

${ }^{2}$ Ch. Binek and B. Doudin, J. Phys.: Condens. Matter 17, L39 (2005).

${ }^{3}$ T. He, Q. Huang, A. P. Ramirez, Y. Wang, K. A. Regan, N. Rogado, M. A. Hayward, M. K. Haas, J. S. Slusky, K. Inumara, H. W. Zandbergen, N. P. Ong, and R. J. Cava, Nature (London) 411, 54 (2001).

${ }^{4}$ K. Kamishima, T. Goto, H. Nakagawa, N. Miura, M. Ohashi, N. Mori, T. Sasaki, and T. Kanomata, Phys. Rev. B 63, 024426 (2000).

${ }^{5}$ K. Takenaka and H. Takagi, Appl. Phys. Lett. 87, 261902 (2005). The lattice contraction with the temperature decrease is very small below the first-order transition while it is large above the transition. Projecting to zero temperatures $\Delta L / L=0.008$.

${ }^{6}$ Magnitude of spin-orbit interactions in considered cubic structures is orders of magnitude smaller than the exchange interactions. SO coupling does not affect the relative orientation of spin moments.

${ }^{7}$ E. O. Chi, Solid State Commun. 120, 307 (2001).

${ }^{8}$ D. Fruchart and E. F. Bertaut, J. Phys. Soc. Jpn. 44, 781 (1978).

${ }^{9}$ P. Blöchl, Phys. Rev. B 50, 17953 (1994).

${ }^{10}$ G. Kresse and D. Joubert, Phys. Rev. B 59, 1758 (1999).

${ }^{11}$ J. P. Perdew, K. Burke, and M. Ernzerhof, Phys. Rev. Lett. 77, 3865 (1996).

${ }^{12}$ D. Hobbs, G. Kresse, and J. Hafner, Phys. Rev. B 62, 11556 (2000); D. Hobbs, J. Hafner, and D. Spike, ibid. 68, 014407 (2003).

${ }^{13}$ P. E. Blöchl, O. Jepsen, and O. K. Andersen, Phys. Rev. B 49,
16223 (1994).

${ }^{14}$ E. F. Bertaut, D. Fruchart, J. P. Bouchaud, and R. Fruchart, Solid State Commun. 6, 251 (1968).

${ }^{15}$ K. H. Kim, K. J. Lee, D. J. Kim, Y. E. Ihm, D. Djayaprawira, M. Takahashi, C. S. Kim, C. G. Kim, and S. H. Yoo, Appl. Phys. Lett. 82, 1775 (2003).

${ }^{16}$ W. S. Kim, E. O. Chi, J. C. Kim, N. H. Hur, K. W. Lee, and Y. N. Choi, Phys. Rev. B 68, 172402 (2003).

${ }^{17}$ J. Kübler, K.-H. Höck, J. Sticht, and A. R. Williams, J. Appl. Phys. 63, 3482 (1988).

${ }^{18}$ E. V. Gomonaj and V. A. L'vov, Phase Transitions 40, 225 (1992).

${ }^{19}$ A. P. Cracknell, Magnetism in Crystalline Materials: Applications of the Theory of Groups of Combiant Symmetry, 1st ed. (Pergamon, Oxford, 1975), pp. 114-116.

${ }^{20}$ J. Ryu, S. Priya, K. Uchino, and H.-E. Kim, J. Electroceram. 8, 107 (2002).

${ }^{21}$ The magnetoelectric voltage coefficient (MEVC) is used more frequently for the composite systems instead of ME coefficient. The MEVC is defined as $\frac{\delta E}{\delta H}$ (see Ref. 19).

${ }^{22}$ Relaxor ferroelectrics such as lead magnesium niobate compounds achieve $2 \%$ strain, about ten times that in conventional ferroelectrics.

${ }^{23}$ F. Zavaliche, H. Zheng, L. Mohaddes-Ardabili, S. Y. Yang, Q. Zhan, P. Shafer, E. Reilly, R. Chopdekar, Y. Jia, P. Wright, D. G. Schlom, Y. Suzuki, and R. Ramesh, Nano Lett. 5, 1793 (2005).

${ }^{24}$ H. S. Choi, W. S. Kim, J. C. Kim, and N. H. Hur, J. Mater. Res. 17, 2640 (2002) 